\title{
POSITIVE GLOBAL SOLUTIONS FOR A GENERAL MODEL OF SIZE-DEPENDENT POPULATION DYNAMICS
}

\author{
NOBUYUKI KATO
}

Received 12 December 2000

We study size-structured population models of general type which have the growth rate depending on the size and time. The local existence and uniqueness of the solution have been shown by Kato and Torikata (1997). Here, we discuss the positivity of the solution and global existence as well as $L^{\infty}$ solutions.

\section{Introduction}

We are concerned with size-structured population models of general type with the growth rate depending on the individual's size and time. In [3], the local existence and uniqueness of the solution have been investigated. In this paper, we discuss the positivity and global existence of the solution as well as $L^{\infty}$ solutions.

As is explained in [3], the model in our mind is the population dynamics of plants in forests or plantations. In this case, the growth rate may be influenced by the environment such as the light, temperature, and nutrients. These must change with time. It is also reasonable to think that the growth rate varies with the size because the size is important to capture the light to grow. From these points, it is natural to consider the growth rate depending on the size and time.

From the mathematical point of view, our results are the generalizations of G. Webb's results [5, Theorems 2.3, 2.4, 2.5, and 4.3] in the age-dependent case. Besides, we investigate $L^{\infty}$ solutions.

Our results also have a close relation to the results of A. Calsina and J. Saldaña [1], where they treated a nonlinear growth rate depending on the size and the total population at each time, whereas the aging and birth functions have the special form of the Gurtin-MacCamy type (see below). For other related works, we refer to [4], where a finite number of structure variables are treated. 
In this paper, we study the following initial boundary value problem with nonlocal boundary condition:

$$
\begin{gathered}
u_{t}+(V(x, t) u)_{x}=G(u(\cdot, t))(x), \quad x \in[0, l), a \leq t \leq T, \\
V(0, t) u(0, t)=C(t)+F(u(\cdot, t)), \quad a \leq t \leq T, \\
u(x, a)=u_{a}(x), \quad x \in[0, l) .
\end{gathered}
$$

The unknown function $u(x, t)$ stands for the density of the population of size $x$ at time $t$, so that the integral $\int_{0}^{l} u(x, t) d x$ represents the total population at time $t$, where $l \in(0, \infty]$ is the maximum size. The function $V$ is the growth rate depending on the size $x$ and time $t$. The mappings $F$ and $G$ correspond to the birth and aging functions, respectively. The typical and important example is the Gurtin-MacCamy type (cf. [2]), that is,

$$
F(\phi)=\int_{0}^{l} \beta(x, P \phi) \phi(x) d x, \quad G(\phi)(x)=-m(x, P \phi) \phi(x),
$$

where $P \phi=\int_{0}^{l} \phi(x) d x$. The function $C$ represents the inflow of zero-size individuals (that is, newborns) from an external source.

In Section 2, we state our assumptions and preliminary results. In Section 3, we study the existence of a positive solution. Section 4 is devoted to study positive $L^{\infty}$ solutions. We discuss the continuability and global existence of the solution in Sections 5 and 6.

\section{Preliminaries}

In this section, we state our general assumptions and the preliminary results. Let $L^{1}:=$ $L^{1}\left(0, l ; \mathbb{R}^{n}\right)$ be the Banach space of Lebesgue integrable functions from $[0, l)$ to $\mathbb{R}^{n}$ with norm $\|f\|_{L^{1}}:=\int_{0}^{l}|f(x)| d x$ for $f \in L^{1}$, where $|\cdot|$ denotes the norm of $\mathbb{R}^{n}$ defined by $|\xi|=\sum_{i=1}^{n}\left|\xi_{i}\right|$ for $\xi=\left(\xi_{1}, \ldots, \xi_{n}\right)$. For $T>a$, let $L_{a, T}:=C\left([a, T] ; L^{1}\right)$ be the Banach space of $L^{1}$-valued continuous functions on $[a, T]$ with the supremum norm $\|u\|_{L_{a, T}}:=\sup _{a \leq t \leq T}\|u(t)\|_{L^{1}}$ for $u \in L_{a, T}$. Note that each element of $L_{a, T}$ is identified with an element of $L^{1}\left((0, l) \times(a, T) ; \mathbb{R}^{n}\right)$ by the relation $[u(t)](x)=u(x, t)$ for $t \in[a, T]$ a.e. $x \in(0, l)$. See [5, Lemma 2.1].

Our general hypotheses are as follows.

$\left(\mathrm{F}_{0}\right) F: L^{1} \rightarrow \mathbb{R}^{n}$ and there is an increasing function $c_{1}:[0, \infty) \rightarrow[0, \infty)$ such that

$$
\left|F\left(\phi_{1}\right)-F\left(\phi_{2}\right)\right| \leq c_{1}(r)\left\|\phi_{1}-\phi_{2}\right\|_{L^{1}}
$$

for all $\phi_{1}, \phi_{2} \in L^{1}$ with $\left\|\phi_{1}\right\|_{L^{1}},\left\|\phi_{2}\right\|_{L^{1}} \leq r$.

$\left(\mathrm{G}_{0}\right) G: L^{1} \rightarrow L^{1}$ and there is an increasing function $c_{2}:[0, \infty) \rightarrow[0, \infty)$ such that

$$
\left\|G\left(\phi_{1}\right)-G\left(\phi_{2}\right)\right\|_{L^{1}} \leq c_{2}(r)\left\|\phi_{1}-\phi_{2}\right\|_{L^{1}}
$$

for all $\phi_{1}, \phi_{2} \in L^{1}$ with $\left\|\phi_{1}\right\|_{L^{1}},\left\|\phi_{2}\right\|_{L^{1}} \leq r$. 
$\left(\mathrm{V}_{0}\right) V:[0, l) \times[0, T] \rightarrow(0, \infty)$ is a bounded function with upper bound $V^{*}$ and $V(l, \cdot)=0$ if $l<\infty . V(x, t)$ is differentiable with respect to $x \in[0, l)$ and there is a Lipschitz constant $L_{V}$ such that

$$
\left|V\left(x_{1}, t\right)-V\left(x_{2}, t\right)\right| \leq L_{V}\left|x_{1}-x_{2}\right|
$$

for all $x_{1}, x_{2} \in[0, l)$ and $t \in[0, T]$. For each $x \in[0, l)$, the mapping $t \mapsto V(x, t)$ is continuous.

$\left(\mathrm{C}_{0}\right) C:[0, T] \rightarrow \mathbb{R}^{n}$ is a continuous function.

Remark 2.1. We require the differentiability of $x \mapsto V(x, t)$ at each $x$ (not only a.e. $x$ ) since it seems necessary even for the previous paper [3, Lemma 3.4]. Thus the partial derivative $V_{x}(x, t)$ satisfies $\left|V_{x}(x, t)\right| \leq L_{V}$ for all $x \in[0, l)$ and $t \in[0, T]$.

In order to define the characteristic curves, we extend the function $V(x, t)$ on $(-\infty, \infty) \times[0, T]$ by defining $V(x, t):=V(0, t)$ for $(x, t) \in(-\infty, 0) \times[0, T]$ and in case $l<\infty, V(x, t):=0$ for $(x, t) \in[l, \infty) \times[0, T]$.

The characteristic curve $\varphi\left(t ; t_{0}, x_{0}\right)$ through $\left(x_{0}, t_{0}\right) \in[0, l) \times[0, T]$ is defined by the unique solution of the differential equation

$$
x^{\prime}(t)=V(x(t), t), \quad t \in\left[t_{0}, T\right]
$$

with the initial condition $x\left(t_{0}\right)=x_{0} \in[0, l)$. Let $z_{a}(t):=\varphi(t ; a, 0)$ denote the characteristic curve through $(0, a)$ in the $(x, t)$-plane.

For $(x, t) \in[0, l) \times[0, T]$ such that $x<z_{0}(t)$, define $\tau:=\tau(t, x)$ implicitly by the relation

$$
\varphi(t ; \tau, 0)=x, \quad \text { or equivalently, } \quad \varphi(\tau ; t, x)=0,
$$

that is, $\tau$ is the initial time of the characteristic through $(x, t)$. Define $\tau_{a}^{*}$ by

$$
\tau_{a}^{*}\left(t_{0}, x_{0}\right)= \begin{cases}\tau\left(t_{0}, x_{0}\right) & \text { for } x_{0}<z_{a}\left(t_{0}\right), \\ a & \text { for } x_{0} \geq z_{a}\left(t_{0}\right) .\end{cases}
$$

Note that the characteristics $x(t)=\varphi\left(t ; t_{0}, x_{0}\right)$ satisfies the integral equation

$$
\varphi\left(t ; t_{0}, x_{0}\right)=x_{0}+\int_{t_{0}}^{t} V\left(\varphi\left(\sigma ; t_{0}, x_{0}\right), \sigma\right) d \sigma \quad \text { for } t \in[0, T],
$$

and that $0 \leq x(t)<l$ for every $t \in\left[\tau_{a}^{*}\left(t_{0}, x_{0}\right), T\right]$ provided that $x_{0} \in[0, l)$. (In case of $l<\infty$, the assumption $V(l, \cdot)=0$ assures $x(t)<l$.)

We define a solution of (1.1) by the analogy of the age-dependent case [5, (1.49)]. See [3, Definition 2.1].

Definition 2.2. A function $u \in L_{a, T}$ is called a solution of (1.1) on $[a, T]$ (with initial value $u_{a}$ ) if $u$ satisfies

$$
u(x, t)= \begin{cases}\frac{\tilde{F}_{\tau}(u(\cdot, \tau))}{V(0, \tau)}+\int_{\tau}^{t} \tilde{G}_{s}(u(\cdot, s))(\varphi(s ; \tau, 0)) d s & \text { a.e. } x \in\left(0, z_{a}(t)\right), \\ u_{a}(\varphi(a ; t, x))+\int_{a}^{t} \tilde{G}_{s}(u(\cdot, s))(\varphi(s ; t, x)) d s & \text { a.e. } x \in\left(z_{a}(t), l\right),\end{cases}
$$


where $\tau:=\tau(t, x)$ is given by (2.5), and for each $t \in[a, T], \tilde{F}_{t}: L^{1} \rightarrow \mathbb{R}^{n}$ and $\tilde{G}_{t}: L^{1} \rightarrow L^{1}$ are given by

$$
\begin{gathered}
\tilde{F}_{t}(\phi):=C(t)+F(\phi), \\
\tilde{G}_{t}(\phi)(x):=G(\phi)(x)-V_{x}(x, t) \phi(x) \quad \text { a.e. } x \in(0, l),
\end{gathered}
$$

for $\phi \in L^{1}$.

Here, we recall some properties of the characteristic curves which are needed in the argument below. For the rest of properties, see Section 3 of [3].

Lemma $2.3\left[3\right.$, Lemmas 3.3, 3.4]. (i) For any $t \in[0, T]$, put $\tau_{t}(x):=\tau(t, x)$. Then $\tau_{t}:\left[0, z_{0}(t)\right] \rightarrow[0, t]$ is continuous, decreasing and onto, and hence $\tau_{t}$ has the inverse $\tau_{t}^{-1}(\cdot)$ which is continuous from $[0, t]$ onto $\left[0, z_{0}(t)\right]$.

(ii) Let $x=\varphi(t ; \tau, \eta)$. Then $x$ is differentiable with respect to $\tau$ and

$$
\frac{d x}{d \tau}=-V(\eta, \tau) \exp \left(\int_{\tau}^{t} V_{x}(\varphi(\sigma ; \tau, \eta), \sigma) d \sigma\right)
$$

and $x$ is differentiable with respect to $\eta$ and

$$
\frac{d x}{d \eta}=\exp \left(\int_{\tau}^{t} V_{x}(\varphi(\sigma ; \tau, \eta), \sigma) d \sigma\right) .
$$

\section{Positive solutions}

In this section, we show the existence of a positive solution of (1.1). Let $L_{+}^{1}:=\{f \in$ $L^{1} \mid f(x) \in \mathbb{R}_{+}^{n}$, a.e. $\left.x \in(0, l)\right\}$, where $\mathbb{R}_{+}^{n}$ is the usual positive cone in $\mathbb{R}^{n}$ and let $L_{a, T,+}:=C\left([a, T] ; L_{+}^{1}\right)$. By a positive solution, we mean the function $u \in L_{a, T,+}$ which satisfies (2.8). We begin with some preliminary lemmas.

Lemma 3.1. For $\alpha \in \mathbb{R}$ and $u \in L_{a, T}$, let

$$
K^{\alpha} u(x, t)= \begin{cases}e^{-\alpha(t-\tau)} \frac{\tilde{F}_{\tau}(u(\cdot, \tau))}{V(0, \tau)} & \\ +\int_{\tau}^{t} e^{-\alpha(t-s)}\left[\tilde{G}_{s}+\alpha I\right](u(\cdot, s))(\varphi(s ; \tau, 0)) d s, & \text { a.e. } x \in\left(0, z_{a}(t)\right), \\ e^{-\alpha(t-a)} u_{a}(\varphi(a ; t, x)) & \\ +\int_{a}^{t} e^{-\alpha(t-s)}\left[\tilde{G}_{s}+\alpha I\right](u(\cdot, s))(\varphi(s ; t, x)) d s, & \text { a.e. } x \in\left(z_{a}(t), l\right),\end{cases}
$$

where $\tau:=\tau(t, x)$ is given by (2.5). Then for fixed $(t, x) \in[0, T] \times[0, l)$, the function

$$
w^{\alpha}(s):=K^{\alpha} u(\varphi(s ; t, x), s), \quad \text { a.e. } s \in\left(\tau_{a}^{*}, T\right)
$$

is differentiable a.e. on $\left(\tau_{a}^{*}, T\right)$ and satisfies

$$
\frac{d}{d s} w^{\alpha}(s)=-\alpha w^{\alpha}(s)+\left(\tilde{G}_{s}+\alpha I\right)(u(\cdot, s))(\varphi(s ; t, x)), \quad \text { a.e. } s \in\left(\tau_{a}^{*}, T\right),
$$

where $\tau_{a}^{*}:=\tau_{a}^{*}(t, x)$ is defined by (2.6). 
Proof. If $\varphi(s ; t, x) \in\left(0, z_{a}(t)\right)$, since $\tau_{a}^{*}(s, \varphi(s ; t, x))=\tau(s, \varphi(s ; t, x))=\tau(s+h$, $\varphi(s+h ; t, x))$, we have

$$
\begin{aligned}
& \frac{1}{h}\left[K^{\alpha} u(\varphi(s+h ; t, x), s+h)-K^{\alpha} u(\varphi(s ; t, x), s)\right] \\
&=\frac{1}{h}\left[K^{\alpha} u(\varphi(s+h ; s, \varphi(s ; t, x)), s+h)-K^{\alpha} u(\varphi(s ; t, x), s)\right] \\
&=\frac{1}{h}\left[e^{-\alpha(s+h-\tau)}-e^{-\alpha(s-\tau)}\right] \frac{\tilde{F}_{\tau}(u(\cdot, \tau))}{V(0, \tau)} \\
&+\frac{1}{h}\left[\int_{\tau}^{s+h} e^{-\alpha(s+h-\eta)}\left[\tilde{G}_{\eta}+\alpha I\right](u(\cdot, \eta))(\varphi(\eta ; \tau, 0)) d \eta\right. \\
&\left.\quad-\int_{\tau}^{s} e^{-\alpha(s-\eta)}\left[\tilde{G}_{\eta}+\alpha I\right](u(\cdot, \eta))(\varphi(\eta ; \tau, 0)) d \eta\right] \\
&=\frac{1}{h}\left[e^{-\alpha h}-1\right] e^{-\alpha(s-\tau)} \frac{\tilde{F}_{\tau}(u(\cdot, \tau))}{V(0, \tau)} \\
& \quad+\frac{1}{h}\left[e^{-\alpha h}-1\right] \int_{\tau}^{s} e^{-\alpha(s-\eta)}\left[\tilde{G}_{\eta}+\alpha I\right](u(\cdot, \eta))(\varphi(\eta ; \tau, 0)) d \eta \\
& \quad+e^{-\alpha h} \frac{1}{h} \int_{s}^{s+h} e^{-\alpha(s-\eta)}\left[\tilde{G}_{\eta}+\alpha I\right](u(\cdot, \eta))(\varphi(\eta ; \tau, 0)) d \eta \\
& \longrightarrow-\alpha K^{\alpha} u(\varphi(s ; t, x), s)+\left[\tilde{G}_{s}+\alpha I\right](u(\cdot, s))(\varphi(s ; t, x))
\end{aligned}
$$

as $h \rightarrow 0$. If $\varphi(s ; t, x) \in\left(z_{a}(t), l\right)$, then noting that $\tau_{a}^{*}(s, \varphi(s ; t, x))=a$ and the relation $\varphi(\eta ; s+h, \varphi(s+h ; t, x))=\varphi(\eta ; t, x)$, we get

$$
\begin{aligned}
\frac{1}{h}\left[K^{\alpha} u\right. & \left.(\varphi(s+h ; t, x), s+h)-K^{\alpha} u(\varphi(s ; t, x), s)\right] \\
= & \frac{1}{h}\left[K^{\alpha} u(\varphi(s+h ; s, \varphi(s ; t, x)), s+h)-K^{\alpha} u(\varphi(s ; t, x), s)\right] \\
= & \frac{1}{h}\left[e^{-\alpha(s+h)}-e^{-\alpha s}\right] u_{a}(\varphi(a ; t, x)) \\
& +\frac{1}{h}\left[\int_{a}^{s+h} e^{-\alpha(s+h-\eta)}\left[\tilde{G}_{\eta}+\alpha I\right](u(\cdot, \eta))(\varphi(\eta ; t, x)) d \eta\right. \\
& \left.\quad-\int_{a}^{s} e^{-\alpha(s-\eta)}\left[\tilde{G}_{\eta}+\alpha I\right](u(\cdot, \eta))(\varphi(\eta ; t, x)) d \eta\right] \\
& \frac{1}{h}\left[e^{-\alpha h}-1\right] e^{-\alpha s} u_{a}(\varphi(a ; t, x)) \\
& +\frac{1}{h}\left[e^{-\alpha h}-1\right] \int_{a}^{s} e^{-\alpha(s-\eta)}\left[\tilde{G}_{\eta}+\alpha I\right](u(\cdot, \eta))(\varphi(\eta ; t, x)) d \eta \\
& +e^{-\alpha h} \frac{1}{h} \int_{s}^{s+h} e^{-\alpha(s-\eta)}\left[\tilde{G}_{\eta}+\alpha I\right](u(\cdot, \eta))(\varphi(\eta ; t, x)) d \eta \\
\longrightarrow & -\alpha K^{\alpha} u(\varphi(s ; t, x), s)+\left[\tilde{G}_{s}+\alpha I\right](u(\cdot, s))(\varphi(s ; t, x))
\end{aligned}
$$

as $h \rightarrow 0$. Thus the result holds. 
196 Size-dependent population dynamics

Lemma 3.2. For $\alpha, \beta \in \mathbb{R}$ and $u \in L_{a, T}$, we have

$$
K^{\beta} u(x, t)=K^{\alpha} u(x, t)+(\alpha-\beta) \int_{\tau_{a}^{*}}^{t} e^{-\beta(t-\eta)}\left[K^{\alpha} u(\varphi(\eta ; t, x), \eta)-u(\varphi(\eta ; t, x), \eta)\right] d \eta
$$

Proof. By Lemma 3.1,

$$
\frac{d}{d s}\left(w^{\beta}(s)-w^{\alpha}(s)\right)=-\beta\left(w^{\beta}(s)-w^{\alpha}(s)\right)+(\alpha-\beta)\left[w^{\alpha}(s)-u(\varphi(s ; t, x), s)\right] .
$$

Since

$$
w^{\beta}\left(\tau_{a}^{*}\right)=w^{\alpha}\left(\tau_{a}^{*}\right)= \begin{cases}\frac{\tilde{F}_{\tau}(u(\cdot, \tau))}{V(0, \tau)} & \text { a.e. } x \in\left(0, z_{a}(t)\right), \\ u_{a}(x) & \text { a.e. } x \in\left(z_{a}(t), l\right),\end{cases}
$$

we have

$$
w^{\beta}(t)-w^{\alpha}(t)=\int_{\tau_{a}^{*}}^{t} e^{-\beta(s-\eta)}(\alpha-\beta)\left[w^{\alpha}(\eta)-u(\varphi(\eta ; t, x), \eta)\right] d \eta .
$$

This shows the desired equality (3.6).

Corollary 3.3. Let $\alpha, \beta \in \mathbb{R}$. Suppose that $u \in L_{a, T}$ and $K^{\alpha} u \in L_{a, T}$. Then $K^{\beta} u \in$ $L_{a, T}$.

Corollary 3.4. Let $\alpha, \beta \in \mathbb{R}$ and $u \in L_{a, T}$. Then $K^{\alpha} u=u$ implies $K^{\beta} u=u$.

Our result concerning the local existence of a positive solution of (1.1) is the following one, which is the generalization of Theorem 2.4 in [5].

TheOREM 3.5. In addition to $\left(F_{0}\right),\left(G_{0}\right)$, and $\left(V_{0}\right)$, we assume the following hypotheses: $\left(\mathrm{F}_{1}\right) F\left(L_{+}^{1}\right) \subset \mathbb{R}_{+}^{n}$.

$\left(\mathrm{G}_{1}\right)$ There is an increasing function $c_{3}:[0, \infty) \rightarrow[0, \infty)$ such that if $r>0$ and $\phi \in L_{+}^{1}$ with $\|\phi\|_{L^{1}} \leq r$, then $G(\phi)+c_{3}(r) \phi \in L_{+}^{1}$.

$\left(\mathrm{C}_{1}\right) C:[0, T] \rightarrow \mathbb{R}_{+}^{n}$ is a continuous function.

Then for each $r>0$, there exists $\delta>0$ such that for the initial value $u_{a} \in L_{+}^{1}$ with $\left\|u_{a}\right\|_{L^{1}} \leq r$, there exists a unique solution $u \in L_{a, \hat{T},+}$ of (1.1) on $[a, \hat{T}]$ with $\hat{T}:=a+\delta$.

Proof. For each $T>a$, set

$$
M_{T}:=\left\{u \in L_{a, T,+} \mid\|u\|_{L_{a, T}} \leq 2 r, u(\cdot, a)=u_{a}\right\} .
$$

For $u \in L_{a, T,+}$ and $\alpha=c_{3}(2 r)+L_{V}$, define $K^{\alpha} u(x, t)$ by (3.1). As is shown in the proof of Theorem 2.1 in [3], $K^{0} u \in L_{a, T}$, so that we find that $K^{\alpha} u \in L_{a, T}$ by Corollary 3.3. Let $C_{T}:=\sup _{a \leq t \leq T}|C(t)|$ for given $T>0$. Similar to the proof of Theorem 2.1 in [3], 
take $\delta_{1}>0$ so small that

$$
e^{L_{V} \delta_{1}}\left[C_{T}+\left(c_{1}(2 r)+c_{2}(2 r)+L_{V}+\alpha\right) \cdot 2 r+|F(0)|+\|G(0)\|_{L^{1}}\right] \delta_{1}+r e^{L_{V} \delta_{1}} \leq 2 r .
$$

Then for $T_{1}=a+\delta_{1}$, we have $\left\|K^{\alpha} u\right\|_{L a, T_{1}} \leq 2 r$. By the hypotheses $\left(\mathrm{F}_{1}\right),\left(\mathrm{G}_{1}\right)$, and $\left(\mathrm{C}_{1}\right)$, we have $K^{\alpha} u(\cdot, t) \in L_{+}^{1}$ and $K^{\alpha} u(\cdot, a)=u_{a}(\cdot)$, so that $K^{\alpha}$ maps $L_{a, T_{1},+}$ into itself. Further, again similar to the proof of Theorem 2.1 in [3], we find that $K^{\alpha}$ is a contraction by taking $\delta_{2}>0$ so small that $e^{L_{V} \delta_{2}}\left[c_{1}(2 r)+c_{2}(2 r)+L_{V}+\alpha\right] \delta_{2}<1$. Thus for $\hat{T}=a+\delta$ with sufficiently small $\delta>0$, there exists a unique function $u \in C\left([a, \hat{T}] ; L_{+}^{1}\right)$ such that $K^{\alpha} u=u$. By Corollary 3.4, we find that this $u$ is a solution of (1.1) on $[a, \hat{T}]$.

\section{4. $L^{\infty}$ solutions}

In this section, we show the existence of a positive $L^{\infty}$ solution of (1.1). Let $L^{\infty}:=$ $L^{\infty}\left(0, l ; \mathbb{R}^{n}\right)$ and for $c \geq 0$, set

$$
D_{c}:=\left\{u \in L_{+}^{1} \cap L^{\infty} \mid\|u\|_{L^{\infty}} \leq c,\|u\|_{L^{1}} \leq c\right\} .
$$

Concerning the $L^{\infty}$ solutions, we obtain the following.

THEOREM 4.1. In addition to $\left(F_{0}\right),\left(G_{0}\right),\left(V_{0}\right),\left(F_{1}\right)$, and $\left(C_{1}\right)$, assume the following hypotheses:

$\left(\mathrm{G}_{2}\right)$ There exist an increasing function $c_{4}:[0, \infty) \rightarrow[0, \infty)$ and a function $c_{5}$ : $[0, \infty) \rightarrow[0, \infty)$ such that $\phi \in D_{r}$ implies $G(\phi)+c_{4}(r) \phi \in D_{c_{5}}(r)$.

Then for each $r>0$, there exists $\delta>0$ such that for $u_{a} \in L_{+}^{1} \cap L^{\infty}$ with $\max \left\{\left\|u_{a}\right\|_{L^{1}}\right.$, $\left.\left\|u_{a}\right\|_{L^{\infty}}\right\} \leq r$, there exists a unique solution $u \in L_{a, \hat{T},+} \cap L^{\infty}\left((a, \hat{T}) \times(0, l) ; \mathbb{R}^{n}\right)$ of (1.1) with $\hat{T}=a+\delta$.

Proof. Let $p(r) \geq 2 \max \left\{r,\left[C_{a, T}+c_{1}(2 r) \cdot 2 r+|F(0)|\right] / V_{a, T}\right\}$, where $C_{a, T}:=$ $\sup _{a \leq t \leq T}|C(t)|$ and $V_{a, T}:=\min _{a \leq t \leq T} V(0, t)>0$ for given $T>a$. For each $T>a$, set

$$
\begin{gathered}
M_{T}^{\infty}:=\left\{u \in L_{a, T,+} \cap L^{\infty}\left((a, T) \times(0, l) ; \mathbb{R}^{n}\right) \mid\|u\|_{L_{a, T}} \leq 2 r,\right. \\
\left.\|u\|_{L^{\infty}\left((a, T) \times(0, l) ; \mathbb{R}^{n}\right)} \leq p(r), u(\cdot, a)=u_{a}\right\} .
\end{gathered}
$$

Note that $M_{T}^{\infty}$ is a closed subset of $L_{a, T}$. For $u \in L_{a, T,+}$ and $\beta:=c_{4}(2 r)+L_{V}$, define $K^{\beta} u(x, t)$ by (3.1). As in the proof of Theorem 3.5, we know that $K^{\beta} u \in L_{a, T,+}$ and for $T_{1}=a+\delta$ with small $\delta>0$ satisfying

$$
e^{L_{V} \delta}\left[C_{a, T}+\left(c_{1}(2 r)+c_{2}(2 r)+L_{V}+\beta\right) \cdot 2 r+|F(0)|+\|G(0)\|_{L^{1}}\right] \delta+r e^{L_{V} \delta} \leq 2 r,
$$

we have $\left\|K^{\beta} u\right\|_{L a, T_{1}} \leq 2 r$. Further, for $T_{2}=a+\delta$ with sufficiently small $\delta>0$ (which may be different from the above one), we show that $u \in M_{T_{2}}^{\infty}$ implies $\left|K^{\beta} u(x, t)\right| \leq$ 
$p(r)$. Indeed, for $x \in\left(0, z_{a}(t)\right)$,

$$
\begin{aligned}
\left|K^{\beta} u(x, t)\right| \leq & e^{-\beta(t-\tau)} \frac{|C(\tau)|+|F(u(\cdot, \tau))|}{V(0, \tau)} \\
& +\int_{\tau}^{t} e^{-\beta(t-s)}\left|\left(G+c_{4}(p(r)) I\right)(u(\cdot, s))(\varphi(s ; \tau, 0))\right| d s \\
& +\int_{\tau}^{t} e^{-\beta(t-s)}\left|\left(L_{V}-V_{x}(\varphi(s ; \tau, 0), s)\right) u(\varphi(s ; \tau, 0), s)\right| d s \\
\leq & \frac{C_{a, T}+c_{1}(2 r) \cdot 2 r+|F(0)|}{V_{a, T}}+\left[c_{5}(p(r))+2 L_{V} \cdot p(r)\right](t-\tau) \\
\leq & \frac{p(r)}{2}+\left[c_{5}(p(r))+2 L_{V} \cdot p(r)\right](T-a),
\end{aligned}
$$

and for $x \in\left(z_{a}(t), l\right)$,

$$
\begin{aligned}
\left|K^{\beta} u(x, t)\right| \leq & e^{-\beta(t-a)}\left|u_{a}(\varphi(a ; t, x))\right| \\
& +\int_{a}^{t} e^{-\beta(t-s)}\left|\left(G+c_{4}(p(r)) I\right)(u(\cdot, s))(\varphi(s ; t, x))\right| d s \\
& +\int_{a}^{t} e^{-\beta(t-s)}\left|\left(L_{V}-V_{x}(\varphi(s ; t, x), s)\right) u(\varphi(s ; t, x), s)\right| d s \\
\leq & r+\left[c_{5}(p(r))+2 L_{V} \cdot p(r)\right](t-a) \\
\leq & \frac{p(r)}{2}+\left[c_{5}(p(r))+2 L_{V} \cdot p(r)\right](T-a) .
\end{aligned}
$$

Hence it is enough to take $\delta>0$ so small that

$$
\frac{p(r)}{2}+\left[c_{5}(p(r))+2 L_{V} \cdot p(r)\right] \delta \leq p(r) .
$$

It is evident that $K^{\beta} u(x, a)=u_{a}(x)$, so we find that $K^{\beta}$ maps $M_{T}^{\infty}$ into itself for $T=a+\delta$ with sufficiently small $\delta>0$.

Similar to the proof of Theorem 2.1 in [3], we find that $K^{\beta}$ is a contraction by taking $\delta>0$ so small that $e^{L_{V} \delta}\left[c_{1}(2 r)+c_{2}(2 r)+L_{V}+\beta\right] \delta<1$. Consequently, for $\hat{T}=a+\delta$ with sufficiently small $\delta>0$, there exists a unique function $u \in M_{\hat{T}}^{\infty}$ such that $K^{\beta} u=u$. By Corollary 3.4, this $u$ is a solution of (1.1).

\section{Continuability and global existence}

By Theorem 2.1 of [3], (1.1) admits a unique local solution for the initial data $u_{0} \in L^{1}$ under the assumptions $\left(\mathrm{F}_{0}\right),\left(\mathrm{G}_{0}\right),\left(\mathrm{V}_{0}\right)$, and $\left(\mathrm{C}_{0}\right)$. Let $\left[0, T_{u_{0}}\right)$ be the maximal interval of existence of the solution, which means that for each $T \in\left(0, T_{u_{0}}\right)$, the solution $u \in L_{0, T}$ of (1.1) on $[0, T]$ with initial value $u_{0}$ exists. The following is the (1.1) version of Theorem 2.3 in [5].

Proposition 5.1. Assume $\left(F_{0}\right),\left(G_{0}\right)$, $\left(V_{0}\right)$, and $\left(C_{0}\right)$ hold. Let $u_{0} \in L^{1}$ and let $u$ be the solution of (1.1) on $\left[0, T_{u_{0}}\right)$. If $T_{u_{0}}<\infty$, then we have $\lim \sup _{t \uparrow T_{u_{0}}}\|u(\cdot, t)\|_{L^{1}}=\infty$. 
Proof. Suppose that $\limsup \operatorname{si\uparrow }_{t u_{0}}\|u(\cdot, t)\|_{L^{1}}<\infty$. Then the norm $\|u(\cdot, t)\|_{L^{1}}$ is bounded, namely, there exists $r>0$ such that $\sup _{0 \leq t<T_{u_{0}}}\|u(\cdot, t)\|_{L^{1}} \leq r$.

By the local existence result due to Theorem 2.1 of [3], there exists some $\delta>0$ depending only on $r$ such that for $0 \leq a<T_{u_{0}}, \hat{u}_{a} \in L^{1}$ with $\left\|\hat{u}_{a}\right\|_{L^{1}} \leq r$, there exists a unique solution $u \in L_{a, a+\delta}$ of (1.1) on $[a, a+\delta]$ with $u(\cdot, a)=\hat{u}_{a}$.

For $a=T_{u_{0}}-\delta / 2$ and $\hat{u}_{a}(\cdot)=u\left(\cdot, T_{u_{0}}-\delta / 2\right)$, since $\left\|\hat{u}_{a}\right\|_{L^{1}} \leq r$, there exists a unique solution $\hat{u} \in L_{T_{u_{0}}-\delta / 2, T_{u_{0}}+\delta / 2}$ of (1.1) on $\left[T_{u_{0}}-\delta / 2, T_{u_{0}}+\delta / 2\right]$. Define

$$
\bar{u}(x, t)= \begin{cases}u(x, t), & 0 \leq t \leq T_{u_{0}}-\frac{\delta}{2}, \\ \hat{u}(x, t), & T_{u_{0}}-\frac{\delta}{2} \leq t \leq T_{u_{0}}+\frac{\delta}{2} .\end{cases}
$$

Then by the uniqueness of the solution (see [3, Theorem 2.2]), we find that this $\bar{u}$ becomes the solution of (1.1) on $\left[0, T_{u_{0}}+\delta / 2\right]$ with the initial data $u_{0}$. But this contradicts the definition of $T_{u_{0}}$.

We look for the condition which assures the global existence of a positive solution of (1.1). The next result is the generalization of Theorem 2.5 in [5].

THEOREM 5.2. Let $\left(F_{0}\right),\left(G_{0}\right),\left(V_{0}\right),\left(F_{1}\right),\left(G_{1}\right)$, and $\left(C_{1}\right)$ hold. Assume further that there exist $\omega_{1} \in \mathbb{R}$ and $\omega_{2} \in \mathbb{R}$ satisfying

$$
\sum_{i=1}^{n}\left[F(\phi)_{i}+\int_{0}^{l} G(\phi)_{i}(x) d x\right] \leq \omega_{1}+\omega_{2} \sum_{i=1}^{n} \int_{0}^{l} \phi_{i}(x) d x, \quad \forall \phi \in L_{+}^{1} .
$$

Then for $u_{0} \in L_{+}^{1}$, we have $T_{u_{0}}=\infty$, that is, there exists a global solution of (1.1) on $[0, \infty)$ with initial value $u_{0}$ and the following estimate holds:

$$
\begin{aligned}
\|u(\cdot, t)\|_{L^{1}} \leq & e^{\left(\omega_{2}+2 L_{V}\right)(t-a)}\|u(\cdot, a)\|_{L^{1}} \\
& +\int_{a}^{t} e^{\left(\omega_{2}+2 L_{V}\right)(t-s)}\left[|C(s)|+\omega_{1}\right] d s, \quad t \geq a(\geq 0) .
\end{aligned}
$$

Proof. Suppose that $T_{u_{0}}<\infty$ and let $0 \leq a<T<T_{u_{0}}$. Put $\alpha:=c_{3}(r)+L_{V}$ with $r=\sup _{0 \leq s<T}\|u(\cdot, s)\|_{L^{1}}$. Let $u_{a}:=u(\cdot, a)$. Then we have

$$
\begin{aligned}
&\|u(\cdot, t)\|_{L^{1}}= \sum_{i=1}^{n} \int_{0}^{l} u_{i}(x, t) d x \\
&=\sum_{i=1}^{n}\left[\int_{0}^{z_{a}(t)} e^{-\alpha(t-\tau)} \frac{C_{i}(\tau)+F_{i}(u(\cdot, \tau))}{V(0, \tau)} d x\right. \\
&+\int_{0}^{z_{a}(t)} \int_{\tau}^{t} e^{-\alpha(t-s)}\left[\left(G+c_{3}(r) I\right)_{i}(u(\cdot, s))(\varphi(s ; \tau, 0))\right. \\
&\left.+\left(L_{V}-V_{x}(\varphi(s ; \tau, 0), s)\right) u_{i}(\varphi(s ; \tau, 0), s)\right] d s d x
\end{aligned}
$$




$$
\begin{aligned}
& +\int_{z_{a}(t)}^{l} e^{-\alpha(t-a)} u_{a i}(\varphi(a ; t, x)) d x \\
& +\int_{z_{a}(t)}^{l} \int_{a}^{t} e^{-\alpha(t-s)}\left[\left(G+c_{3}(r) I\right)_{i}(u(\cdot, s))(\varphi(s ; t, x))\right. \\
& \left.\left.\quad+\left(L_{V}-V_{x}(\varphi(s ; t, x), s)\right) u_{i}(\varphi(s ; t, x), s)\right] d s d x\right] \\
& =: \sum_{i=1}^{n}\left[I_{1}^{i}+I_{2}^{i}+I_{3}^{i}+I_{4}^{i}\right] .
\end{aligned}
$$

For $I_{1}^{i}$, by the change of variable $\xi=\tau(t, x)$, we have

$$
\begin{aligned}
I_{1}^{i} & =\int_{a}^{t} e^{-\alpha(t-\xi)}\left[C_{i}(\xi)+F_{i}(u(\cdot, \xi))\right] e^{\int_{\xi}^{t} V_{x}(\varphi(\sigma ; \xi, 0), \sigma) d \sigma} d \xi \\
& \leq \int_{a}^{t} e^{-\alpha(t-\xi)}\left[C_{i}(\xi)+F_{i}(u(\cdot, \xi))\right] e^{L_{V}(t-\xi)} d \xi \\
& =\int_{a}^{t} e^{-c_{3}(r)(t-\xi)}\left[C_{i}(\xi)+F_{i}(u(\cdot, \xi))\right] d \xi .
\end{aligned}
$$

Next, $I_{2}^{i}+I_{4}^{i}$ is estimated as follows:

$$
\begin{aligned}
& I_{2}^{i}+I_{4}^{i}=\int_{a}^{t} \int_{\tau_{t}^{-1}(s)}^{z_{a}(t)} e^{-\alpha(t-s)}\left[\left(G+c_{3}(r) I\right)_{i}(u(\cdot, s))(\varphi(s ; \tau, 0))\right.\left.+\left(L_{V}-V_{x}(\varphi(s ; \tau, 0), s)\right) u_{i}(\varphi(s ; \tau, 0), s)\right] d x d s \\
&+\int_{a}^{t} \int_{z_{a}(t)}^{l} e^{-\alpha(t-s)}\left[\left(G+c_{3}(r) I\right)_{i}(u(\cdot, s))(\varphi(s ; t, x))\right. \\
&\left.\quad+\left(L_{V}-V_{x}(\varphi(s ; t, x), s)\right) u_{i}(\varphi(s ; t, x), s)\right] d x d s \\
&=\int_{a}^{t} \int_{0}^{l} e^{-\alpha(t-s)}\left[\left(G+c_{3}(r) I\right)_{i}(u(\cdot, s))(\eta)+\left(L_{V}-V_{x}(\eta, s)\right) u_{i}(\eta, s)\right] \\
& \leq \int_{a}^{t} \int_{0}^{l} e^{-c_{3}(r)(t-s)}\left[\left(G+c_{3}(r) I\right)_{i}(u(\cdot, s))(\eta)+\left(L_{V}-V_{x}(\eta, s)\right) u_{i}(\eta, s)\right] d \eta d s .
\end{aligned}
$$

Here we have used the notation $\tau_{t}^{-1}(s)$ as the inverse of the decreasing function $x \mapsto$ $\tau(t, x)$ for fixed $t$ (see [3, Section 3]). For $I_{3}^{i}$, we have

$$
\begin{aligned}
I_{3}^{i} & =\int_{0}^{l} e^{-\alpha(t-a)} u_{a i}(\eta) e^{\int_{a}^{t} V_{x}(\varphi(\sigma ; a, \eta), \sigma) d \sigma} d \eta \\
& \leq \int_{0}^{l} e^{-\left(\alpha-L_{V}\right)(t-a)} u_{a i}(\eta) d \eta=\int_{0}^{l} e^{-c_{3}(r)(t-a)} u_{a i}(\eta) d \eta
\end{aligned}
$$


Therefore, using the hypothesis (5.2), we have

$$
\begin{aligned}
\|u(\cdot, t)\|_{L^{1}} \leq & \sum_{i=1}^{n}\left[\int_{a}^{t} e^{-c_{3}(r)(t-s)}\left[C_{i}(s)+F_{i}(u(\cdot, s))\right] d s\right. \\
& +\int_{a}^{t} \int_{0}^{l} e^{-c_{3}(r)(t-s)}\left[\left(G+c_{3}(r) I\right)_{i}(u(\cdot, s))(\eta)\right. \\
& \left.+\left(L_{V}-V_{x}(\eta, s)\right) u_{i}(\eta, s)\right] d \eta d s \\
& \left.+\int_{0}^{l} e^{-c_{3}(r)(t-a)} u_{a i}(\eta) d \eta\right] \\
\leq & \int_{a}^{t} e^{-c_{3}(r)(t-s)} \sum_{i=1}^{n} C_{i}(s) d s+e^{-c_{3}(r)(t-a)} \int_{0}^{l} \sum_{i=1}^{n} u_{a i}(\eta) d \eta \\
& +\int_{a}^{t} e^{-c_{3}(r)(t-s)} \sum_{i=1}^{n}\left[F_{i}(u(\cdot, s))+\int_{0}^{l} G_{i}(u(\cdot, s))(\eta) d \eta\right] d s \\
& +\int_{a}^{t} e^{-c_{3}(r)(t-s)} \int_{0}^{l}\left(c_{3}(r)+L_{V}-V_{x}(\eta, s)\right) \sum_{i=1}^{n} u_{i}(\eta, s) d \eta d s \\
\leq & \int_{a}^{t} e^{-c_{3}(r)(t-s)}|C(s)| d s+e^{-c_{3}(r)(t-a)}\left\|_{a}\right\|_{L^{1}} \\
& +\int_{a}^{t} e^{-c_{3}(r)(t-s)}\left[\omega_{1}+\omega_{2} \sum_{i=1}^{n} \int_{0}^{l} u_{i}(\eta, s) d \eta\right] d s \\
& +\left(c_{3}(r)+2 L_{V}\right) \int_{a}^{t} e^{-c_{3}(r)(t-s)}\|u(\cdot, s)\|_{L^{1}} d s .
\end{aligned}
$$

Hence,

$$
\begin{aligned}
e^{c_{3}(r) t}\|u(\cdot, t)\|_{L^{1}} \leq & \int_{a}^{t} e^{c_{3}(r) s}\left[|C(s)|+\omega_{1}\right] d s+e^{c_{3}(r) a}\left\|u_{a}\right\|_{L^{1}} \\
& +\left(\omega_{2}+2 L_{V}+c_{3}(r)\right) \int_{a}^{t} e^{c_{3}(r) s}\|u(\cdot, s)\|_{L^{1}} d s .
\end{aligned}
$$

By Gronwall's lemma, we have

$$
\begin{aligned}
e^{c_{3}(r) t}\|u(\cdot, t)\|_{L^{1}} \leq & \int_{a}^{t} e^{c_{3}(r) s} e^{\left(\omega_{2}+2 L_{V}+c_{3}(r)\right)(t-s)}|C(s)| d s \\
& +e^{c_{3}(r) a} e^{\left(\omega_{2}+2 L_{V}+c_{3}(r)\right)(t-a)}\left\|u_{a}\right\|_{L^{1}} .
\end{aligned}
$$

Rearranging this, we obtain

$$
\|u(\cdot, t)\|_{L^{1}} \leq e^{\left(\omega_{2}+2 L_{V}\right)(t-a)}\left\|u_{a}\right\|_{L^{1}}+\int_{a}^{t} e^{\left(\omega_{2}+2 L_{V}\right)(t-s)}\left[|C(s)|+\omega_{1}\right] d s
$$

for $0 \leq a \leq t \leq T$. Since $T \in\left(0, T_{u_{0}}\right)$ is arbitrary, we obtain the desired estimate (5.3) for $a \leq t<T_{u_{0}}$. From this, we find that $\lim \sup _{t \uparrow T_{u_{0}}}\|u(\cdot, t)\|_{L^{1}}<\infty$, which contradicts the result of Proposition 5.1, and hence we conclude $T_{u_{0}}=\infty$ and (5.3) holds. 
Note that we do not infer from the estimate (5.3) that the norm of the solution is bounded. The next theorem shows the condition for the solution to be $L^{1}$-bounded globally. It is the generalization of Theorem 4.3 in [5].

Theorem 5.3. Let $\left(F_{0}\right),\left(G_{0}\right),\left(V_{0}\right),\left(F_{1}\right),\left(G_{1}\right)$, and $\left(C_{1}\right)$ hold. Assume that $C \in L^{\infty}$ $\left(0, \infty ; \mathbb{R}^{n}\right)$ and there exists $K>0$ such that

$$
\begin{aligned}
& \sum_{i=1}^{n}\left[F(\phi)_{i}+\int_{0}^{l} G(\phi)_{i}(x) d x\right]+\|C\|_{L^{\infty}\left(0, \infty ; \mathbb{R}^{n}\right)} \\
& \quad \leq-L_{V} \sum_{i=1}^{n} \int_{0}^{l} \phi_{i}(x), d x, \quad \forall \phi \in L_{+}^{1},\|\phi\|_{L^{1}} \geq K .
\end{aligned}
$$

Then for $u_{0} \in L_{+}^{1}$, we have $T_{u_{0}}=\infty$, that is, there exists a global solution of (1.1) on $[0, \infty)$ with initial value $u_{0}$ and the following estimate holds:

$$
\sup _{t \geq 0}\|u(\cdot, t)\|_{L^{1}} \leq \max \left\{K,\left\|u_{0}\right\|_{L^{1}}\right\} .
$$

Proof. If (5.13) holds, then $T_{u_{0}}=\infty$ by Proposition 5.1. Consider the case $\left\|u_{0}\right\|_{L^{1}} \leq K$ and suppose the conclusion does not hold. Then there exists $t \in\left(0, T_{u_{0}}\right)$ and $\varepsilon>0$ such that $\|u(\cdot, t)\|_{L^{1}}>K+\varepsilon$. Let $t_{0}:=\inf \left\{t \in\left[0, T_{u_{0}}\right):\|u(\cdot, t)\|_{L^{1}}>K+\varepsilon\right\}$. Since the mapping $t \mapsto\|u(\cdot, t)\|_{L^{1}}$ is continuous from $\left[0, T_{u_{0}}\right)$ to $[0, \infty)$, we have $\left\|u\left(\cdot, t_{0}\right)\right\|_{L^{1}} \geq$ $K+\varepsilon>K$. Hence there exists $r>0$ such that $\|u(\cdot, t)\|_{L^{1}}>K$ for $t \in\left[t_{0}, t_{0}+r\right)$. Similar to the proof of Theorem 5.2, we use (5.12) instead of (5.2) to conclude that

$$
\|u(\cdot, t)\|_{L^{1}} \leq\left\|u\left(\cdot, t_{0}\right)\right\|_{L^{1}}, \quad t \in\left[t_{0}, t_{0}+r\right) .
$$

Assume that $t_{0}>0$. Then we have $\left\|u\left(\cdot, t_{0}\right)\right\|_{L^{1}}=K+\varepsilon$. Thus $\|u(\cdot, t)\|_{L^{1}} \leq K+\varepsilon$ for $t \in\left[t_{0}, t_{0}+r\right)$. This contradicts the definition of $t_{0}$, and so $t_{0}=0$. But then, $K+\varepsilon \leq$ $\left\|u\left(\cdot, t_{0}\right)\right\|_{L^{1}}=\left\|u_{0}\right\|_{L^{1}} \leq K$, which is a contradiction. Hence (5.13) holds.

Next, consider the case $\left\|u_{0}\right\|_{L^{1}}>K$. Assume that there exists $t \in\left(0, T_{u_{0}}\right)$ such that $\|u(\cdot, t)\|_{L^{1}}>\left\|u_{0}\right\|_{L^{1}}$. Let $t_{0}:=\inf \left\{t \in\left(0, T_{u_{0}}\right):\|u(\cdot, t)\|_{L^{1}}>\left\|u_{0}\right\|_{L^{1}}\right\}$. Then $\left\|u\left(\cdot, t_{0}\right)\right\|_{L^{1}}=\left\|u_{0}\right\|_{L^{1}}$. Similar argument as above shows that there exists $r>0$ such that

$$
\|u(\cdot, t)\|_{L^{1}} \leq\left\|u\left(\cdot, t_{0}\right)\right\|_{L^{1}}, \quad t \in\left[t_{0}, t_{0}+r\right) .
$$

This contradicts the definition of $t_{0}$, and hence (5.13) holds.

Remark 5.4. We note that the solution of (1.1) defines an evolution operator in $L^{1}$. Suppose that for each $\phi \in L^{1}, T_{\phi}=\infty$. Let $u \in C\left([s, \infty) ; L^{1}\right)$ be the solution of (1.1) on $[s, \infty)$ with $u(\cdot, s)=\phi$. Then putting

$$
[U(t, s) \phi](x):=u(x, t)
$$

defines a family $\{U(t, s) \mid 0 \leq s \leq t\}$ of nonlinear operators. Recall that Theorem 2.2 in [3] tells that

$$
\|U(t, s) \phi-U(t, s) \hat{\phi}\|_{L^{1}} \leq \exp \left[\left(c_{1}(r)+c_{2}(r)+2 L_{V}\right)(t-s)\right]\|\phi-\hat{\phi}\|_{L^{1}},
$$


where $\|\phi\|_{L^{1}},\|\hat{\phi}\|_{L^{1}} \leq r$. This estimate implies the continuity of $\phi \mapsto U(t, s) \phi$ as well as the uniqueness of the solution which assures the evolution property $U(t, s) U(s, \tau)=$ $U(t, \tau)$ for $0 \leq \tau \leq s \leq t . U(s, s)=I$ is obvious. The continuity $t \mapsto U(t, s) \phi$ from $[s, \infty)$ into $L^{1}$ for each $s \geq 0$ and $\phi \in L^{1}$ follows from the fact that $u \in C\left([s, \infty) ; L^{1}\right)$. Thus $\{U(t, s) \mid 0 \leq s \leq t\}$ becomes a family of nonlinear evolution operators in $L^{1}$.

\section{Global existence for $L^{\infty}$ solution}

We discuss the continuability of $L^{\infty}$ solution. Suppose that all the hypotheses in Theorem 4.1 are satisfied. Let $\left[0, T_{u_{0}}^{*}\right)$ be the maximal interval of existence of the solution $u \in L_{0, T,+} \cap L^{\infty}\left((0, T) \times(0, l) ; \mathbb{R}^{n}\right)\left(\forall T \in\left(0, T_{u_{0}}^{*}\right)\right)$ of (1.1) with initial value $u_{0} \in L_{+}^{1} \cap L^{\infty}$.

Note that $T_{u_{0}}^{*} \leq T_{u_{0}}$, where $T_{u_{0}}$ is the maximal existing time for the solution belonging to $L_{0, T,+}$ (as defined in Section 5).

Proposition 6.1. Assume the hypotheses in Theorem 4.1. If $T_{u_{0}}^{*}<\infty$, then either $\limsup \operatorname{su}_{t \uparrow T_{u_{0}}^{*}}\|u(\cdot, t)\|_{L^{1}}=\infty$ or $\lim \sup _{t \uparrow T_{u_{0}}^{*}}\|u(\cdot, t)\|_{L^{\infty}}=\infty$ holds.

Proof. Suppose for contradiction that both $\limsup _{t \uparrow T_{u_{0}}^{*}}\|u(\cdot, t)\|_{L^{1}}<\infty$ and $\limsup \operatorname{su}_{t T_{u_{0}}^{*}}\|u(\cdot, t)\|_{L^{\infty}}<\infty$ hold. Then there exists some $r>0$ such that $\sup _{0 \leq t<T_{u_{0}}^{*}}\|u(\cdot, t)\|_{L^{1}} \leq r$ and $\sup _{0 \leq t<T_{u_{0}}^{*}}\|u(\cdot, t)\|_{L^{\infty}} \leq r$. By Theorem 4.1, there exists $\delta>0$ depending only on $r$ such that for $0 \leq a<T_{u_{0}}^{*}, \hat{u}_{a} \in L_{+}^{1} \cap L^{\infty}$ with $\left\|\hat{u}_{a}\right\|_{L^{1}} \leq r$ and $\left\|\hat{u}_{a}\right\|_{L^{\infty}} \leq r$, there exists a unique solution $u \in L_{a, a+\delta,+} \cap L^{\infty}((a, a+$ $\left.\delta) \times(0, l) ; \mathbb{R}^{n}\right)$ of $(1.1)$ on $[a, a+\delta]$ with $u(\cdot, a)=\hat{u}_{a}$.

For $a=T_{u_{0}}^{*}-\delta / 2$ and $\hat{u}_{a}(\cdot)=u\left(\cdot, T_{u_{0}}^{*}-\delta / 2\right)$, since $\left\|\hat{u}_{a}\right\|_{L^{1}} \leq r$ and $\left\|\hat{u}_{a}\right\|_{L^{\infty}} \leq r$, there exists a unique solution $\hat{u} \in L_{T_{u_{0}}^{*}-\delta / 2, T_{u_{0}}^{*}+\delta / 2}$ of (1.1) on $\left[T_{u_{0}}^{*}-\delta / 2, T_{u_{0}}^{*}+\delta / 2\right]$. Define

$$
\bar{u}(x, t)= \begin{cases}u(x, t), & 0 \leq t \leq T_{u_{0}}^{*}-\frac{\delta}{2}, \\ \hat{u}(x, t), & T_{u_{0}}^{*}-\frac{\delta}{2} \leq t \leq T_{u_{0}}^{*}+\frac{\delta}{2} .\end{cases}
$$

Then we find that $\bar{u}$ belongs to $u \in L_{a, a+\delta,+} \cap L^{\infty}\left((a, a+\delta) \times(0, l) ; \mathbb{R}^{n}\right)$ and, by the uniqueness of the solution (see [3, Theorem 2.2]), $\bar{u}$ becomes the solution of (1.1) on $\left[0, T_{u_{0}}^{*}+\delta / 2\right]$ with the initial data $u_{0}$. But this contradicts the definition of $T_{u_{0}}^{*}$.

Corollary 6.2. Assume the hypotheses in Theorem 4.1. Suppose either the hypotheses in Theorem 5.2 or those in Theorem 5.3 hold. Then $T_{u_{0}}^{*}<\infty$ implies that $\lim \sup _{t \uparrow T_{u_{0}}^{*}}\|u(\cdot, t)\|_{L^{\infty}}=\infty$.

Theorem 6.3. Assume all the hypotheses in Theorems 4.1 and 5.2 are satisfied. In addition, assume the following hypothesis on $G$ :

$\left(\mathrm{G}_{3}\right)$ there exists a function $c_{6}:[0, \infty) \rightarrow \mathbb{R}$ such that for $\phi \in L_{+}^{1} \cap L^{\infty}$ with $\|\phi\|_{L^{1}} \leq r$, we have $c_{6}(r) \phi-G(\phi) \in L_{+}^{1}$. 
204 Size-dependent population dynamics

Then for each $u_{0} \in L_{+}^{1} \cap L^{\infty}$, we have $T_{u_{0}}^{*}=\infty$ and for each $T \geq 0$ the following estimate holds:

$$
\sup _{0 \leq t \leq T}\|u(\cdot, t)\|_{L^{\infty}} \leq e^{\left(L_{V}+c_{6}\left(r_{T}\right)\right) T} \max \left\{R_{T},\left\|u_{0}\right\|_{L^{\infty}}\right\},
$$

where $r_{T}:=\sup _{0 \leq t \leq T}\|u(\cdot, t)\|_{L^{1}}$ and $R_{T}:=\left[C_{0, T}+c_{1}\left(r_{T}\right) r_{T}+|F(0)|\right] / V_{0, T}\left(C_{0, T}:=\right.$ $\sup _{0 \leq t \leq T}|C(t)|$ and $V_{0, T}:=\min _{0 \leq t \leq T} V(0, t)$ are the ones appeared in the proof of Theorem 4.1).

Proof. Suppose that $T_{u_{0}}^{*}<\infty$. For each $T \in\left(0, T_{u_{0}}^{*}\right)$, we set $\beta:=c_{6}\left(r_{T}\right)$. Let $0 \leq t \leq T$. Recall that by virtue of Corollary 3.4, the solution satisfies $K^{\beta} u=u$, where $K^{\beta}$ is defined by (3.1). Then, for a.e. $x \in\left(0, z_{0}(t)\right)$, we have

$$
\begin{aligned}
|u(x, t)|= & \sum_{i=1}^{n} u_{i}(x, t) \\
= & \sum_{i=1}^{n}\left\{\frac{e^{\beta(t-\tau)}\left[C_{i}(\tau)+F_{i}(u(\cdot, \tau))\right]}{V(0, \tau)} d x\right. \\
& \quad+\int_{\tau}^{t} e^{\beta(t-s)}\left[\left(G-c_{6}\left(r_{T}\right) I\right)_{i}(u(\cdot, s))(\varphi(s ; \tau, 0))\right] d s \\
& \left.\quad-\int_{\tau}^{t} e^{\beta(t-s)} V_{x}(\varphi(s ; \tau, 0), s) u_{i}(\varphi(s ; \tau, 0), s) d s\right\} \\
\leq & e^{\beta(t-\tau)} \frac{\left[C_{0, T}+\sum_{i=1}^{n}\left[F_{i}(u(\cdot, \tau))-F_{i}(0)\right]+|F(0)|\right]}{V_{0, T}} \\
& +L_{V} \int_{\tau}^{t} e^{\beta(t-s)} \sum_{i=1}^{n} u_{i}(\varphi(s ; \tau, 0), s) d s \quad\left(\mathrm{by}\left(G_{3}\right)\right) \\
\leq & \frac{e^{\beta(t-\tau)}\left[C_{0, T}+c_{1}\left(r_{T}\right) r_{T}+|F(0)|\right]}{V_{0, T}}+L_{V} \int_{\tau}^{t} e^{\beta(t-s)}\|u(\cdot, s)\|_{L^{\infty} d s .}
\end{aligned}
$$

For a.e. $x \in\left(z_{0}(t), l\right)$, we have

$$
\begin{aligned}
&|u(x, t)|= \sum_{i=1}^{n} u_{i}(x, t) \\
&=\sum_{i=1}^{n}\left\{e^{\beta t} u_{0 i}(\varphi(0 ; t, x))+\int_{0}^{t} e^{\beta(t-s)}\left[\left(G-c_{6}\left(r_{T}\right) I\right)_{i}(u(\cdot, s))(\varphi(s ; t, x))\right] d s\right. \\
&\left.\quad-\int_{0}^{t} e^{\beta(t-s)} V_{x}(\varphi(s ; t, x), s) u_{i}(\varphi(s ; t, x), s) d s\right\} \\
& \leq e^{\beta t}\left\|u_{0}\right\|_{L^{\infty}}+L_{V} \int_{0}^{t} e^{\beta(t-s)}\|u(\cdot, s)\|_{L^{\infty}} d s .
\end{aligned}
$$


Therefore, we obtain

$$
e^{-\beta t}\|u(\cdot, t)\|_{L^{\infty}} \leq \max \left\{R_{T},\left\|u_{0}\right\|_{L^{\infty}}\right\}+L_{V} \int_{0}^{t} e^{-\beta s}\|u(\cdot, s)\|_{L^{\infty}} d s .
$$

By Gronwall's lemma, we have $\|u(\cdot, t)\|_{L^{\infty}} \leq e^{\left[L_{V}+c_{6}\left(r_{T}\right)\right] t} \max \left\{R_{T},\left\|u_{0}\right\|_{L^{\infty}}\right\}$. Then we obtain $\lim \sup _{t \uparrow T_{u_{0}}^{*}}\|u(\cdot, t)\|_{L^{\infty}}<\infty$. This contradicts Corollary 6.2, and hence, $T_{u_{0}}^{*}=\infty$ and the estimate (6.2) holds.

The following theorem shows the global boundedness of $L^{\infty}$-norm of the solution.

THEOREM 6.4. Assume all the hypotheses in Theorems 4.1 and 5.3 are satisfied. Assume $\left(G_{3}\right)$ holds with $c_{6}$ such that $c_{6}(r) \leq-L_{V}$ for $r \in[0, \infty)$. In addition, assume the following condition:

$\left(\mathrm{V}_{1}\right) \quad V(0, t) \geq V_{*}$ for some $V_{*}>0$.

Then for each $u_{0} \in L_{+}^{1} \cap L^{\infty}$, we have $T_{u_{0}}^{*}=\infty$ and the following estimate holds:

$$
\sup _{0 \leq t<\infty}\|u(\cdot, t)\|_{L^{\infty}} \leq \max \left\{R,\left\|u_{0}\right\|_{L^{\infty}}\right\}
$$

where $R:=\left[\|C\|_{L^{\infty}\left(0, \infty ; \mathbb{R}^{n}\right)}+c_{1}(r) r+|F(0)|\right] / V_{*}$ and $r:=\max \left\{K,\left\|u_{0}\right\|_{L^{1}}\right\}$ with $K$ in (5.12).

Proof. By Theorem 5.3, $\sup _{0 \leq t<\infty}\|u(\cdot, t)\|_{L^{1}} \leq r$. Then the same argument as in Theorem 6.3 leads to $T_{u_{0}}^{*}=\infty$ and the desired estimate (6.6).

\section{Acknowledgements}

The author would like to thank the referee for valuable comments. The referee pointed out an improvement in the previous version of Theorem 5.2, where only the case $\omega_{1}=0$ was considered and directed me to the article [4].

This research was supported in part by Grant-in-Aid for Scientific Research (C)(2), No. 10640172.

\section{References}

[1] A. Calsina and J. Saldaña, A model of physiologically structured population dynamics with a nonlinear individual growth rate, J. Math. Biol. 33 (1995), no. 4, 335-364. MR 96i:92020. Zbl 828.92025.

[2] M. E. Gurtin and R. C. MacCamy, Non-linear age-dependent population dynamics, Arch. Rational Mech. Anal. 54 (1974), 281-300. MR 50\#6550. Zbl 286.92005.

[3] N. Kato and H. Torikata, Local existence for a general model of size-dependent population dynamics, Abstr. Appl. Anal. 2 (1997), no. 3-4, 207-226. MR 2001a:92033. Zbl 931.92024.

[4] S. L. Tucker and S. O. Zimmerman, A nonlinear model of population dynamics containing an arbitrary number of continuous structure variables, SIAM J. Appl. Math. 48 (1988), no. 3, 549-591. MR 89h:92056. Zbl 657.92011. 
206 Size-dependent population dynamics

[5] G. F. Webb, Theory of Nonlinear Age-dependent Population Dynamics, Monographs and Text books in Pure and Applied Mathematics, vol. 89, Marcel Dekker, New York, 1985. MR 86e:92032. Zbl 555.92014.

Nobuyuki Kato: Department of Mathematics, Shimane University, Matsue, Shimane 690-8504, JAPAN

E-mail address: nkato@math.shimane-u.ac.jp 


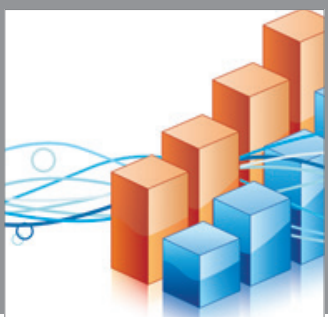

Advances in

Operations Research

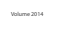

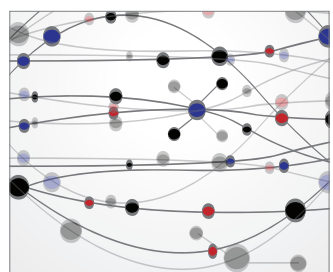

\section{The Scientific} World Journal
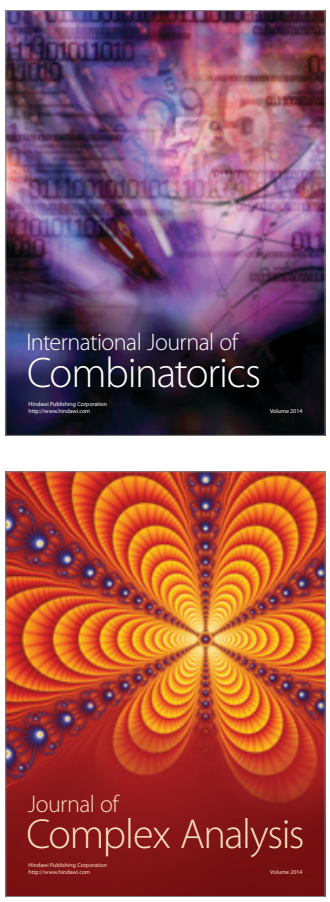

International Journal of

Mathematics and

Mathematical

Sciences
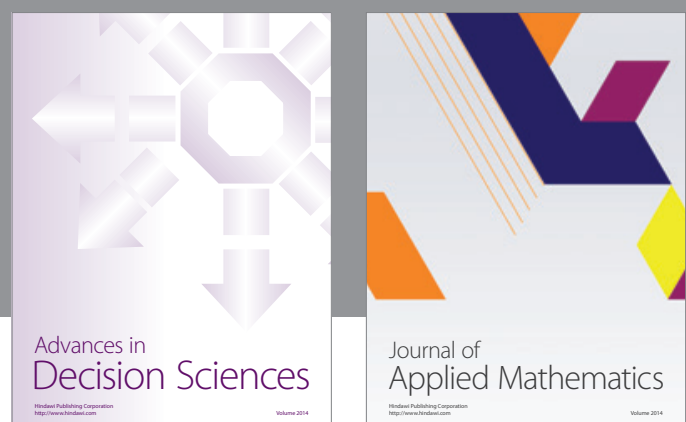

Journal of

Applied Mathematics
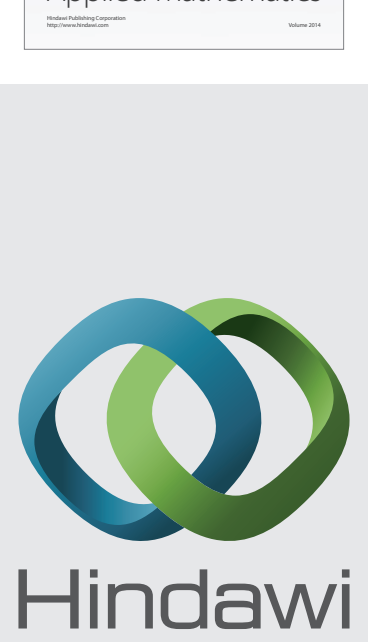

Submit your manuscripts at http://www.hindawi.com
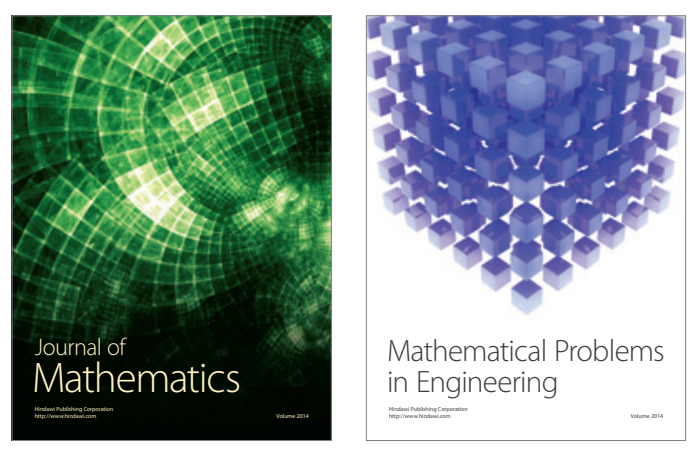

Mathematical Problems in Engineering
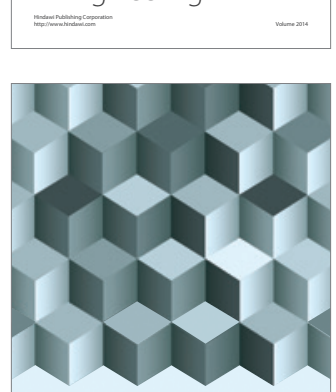

Journal of

Function Spaces
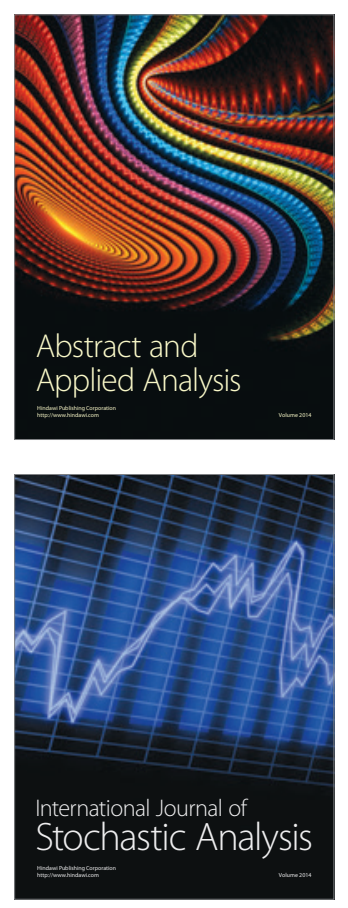

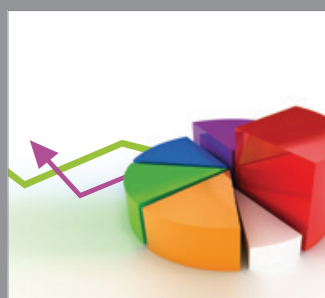

ournal of

Probability and Statistics

Promensencen
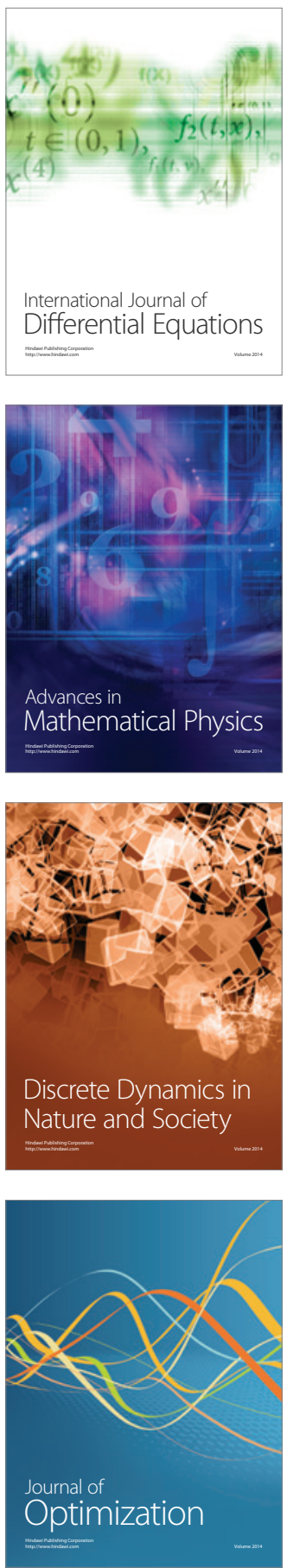